\title{
Stability result of a viscoelastic plate equation with past history and a logarithmic nonlinearity
}

\section{Adel M. Al-Mahdi ${ }^{1 *}$ (D)}

"Correspondence:

almahdi@kfupm.edu.sa

${ }^{1}$ The Preparatory Year Math

Program, King Fahd University of

Petroleum and Minerals, Dhahran,

Saudi Arabia

\section{Springer}

\begin{abstract}
In this paper, we are concerned with the decay rate of the solution of a viscoelastic plate equation with infinite memory and logarithmic nonlinearity. We establish an explicit and general decay rate results with imposing a minimal condition on the relaxation function. In fact, we assume that the relaxation function $h$ satisfies$$
h^{\prime}(t) \leq-\xi(t) H(h(t)), \quad t \geq 0,
$$

where the functions $\xi$ and $H$ satisfy some conditions. Our proof is based on the multiplier method, convex properties, logarithmic inequalities, and some properties of integro-differential equations. Moreover, we drop the boundedness assumption on the history data, usually made in the literature. In fact, our results generalize, extend, and improve earlier results in the literature.
\end{abstract}

MSC: 35B35; 35L55; 75D05; 74D10; 93D20

Keywords: Stability; Logarithmic Sobolev inequalities; Infinite memory; Plate equation; Convexity

\section{Introduction}

In this work, we consider the following viscoelastic plate problem with velocity-dependent material density and logarithmic nonlinearity:

$$
\left|u_{t}\right|^{\rho} u_{t t}+\Delta^{2} u+\Delta^{2} u_{t t}-\int_{0}^{+\infty} h(s) \Delta^{2} u(t-s) d s=\alpha u \ln |u| \quad \text { in } \Omega \times(0, \infty)
$$

equipped with initial and boundary conditions

$$
\begin{aligned}
& u(x, t)=\frac{\partial u}{\partial n}(x, t)=0 \quad \text { in } \partial \Omega \times(0, \infty), \\
& u(x,-t)=u_{0}(x, t), \quad u_{t}(x, 0)=u_{1}(x) \quad \text { in } \Omega,
\end{aligned}
$$

where $\Omega$ is a bounded domain of $\mathbb{R}^{2}$ with smooth boundary $\partial \Omega, n$ is the unit outer normal to $\partial \Omega$, and $\rho$ and $\alpha$ are positive constants. The relaxation function $h$ satisfies the following

(c) The Author(s) 2020. This article is licensed under a Creative Commons Attribution 4.0 International License, which permits use, sharing, adaptation, distribution and reproduction in any medium or format, as long as you give appropriate credit to the original author(s) and the source, provide a link to the Creative Commons licence, and indicate if changes were made. The images or other third party material in this article are included in the article's Creative Commons licence, unless indicated otherwise in a credit line to the material. If material is not included in the article's Creative Commons licence and your intended use is not permitted by statutory regulation or exceeds the permitted use, you will need to obtain permission directly from the copyright holder. To view a copy of this licence, visit http://creativecommons.org/licenses/by/4.0/. 
general condition:

$$
h^{\prime}(t) \leq-\xi(t) H(h(t))
$$

where the functions $\xi$ and $H$ satisfy some conditions specified later. To motivate our work, let us recall some results regarding problems with logarithmic nonlinearity.

\subsection{Problems with logarithmic nonlinearity}

The logarithmic nonlinearity has many applications in physics such as nuclear physics, optics, and geophysics [1-3]. For the problems with logarithmic nonlinearity, we start with the works of Birula and Mycielski [4] and [5], where they proved that the wave equations with logarithmic nonlinearity have stable and localized solutions. Cazenave and Haraux [6] considered the Cauchy problem

$$
u_{t t}-\Delta u=u \ln |u|^{\alpha}
$$

in $\mathbb{R}^{3}$ and established the existence and uniqueness of the solution. The corresponding one-dimensional problem of (4) was studied by Gorka [1], who established the global existence of weak solutions, provided that $\left(u_{0}, u_{1}\right) \in H_{0}^{1} \times L^{2}$. Bartkowski and Gorka [2] investigated weak solutions and also proved the existence of classical solutions. Hiramatsu et al. [3] considered the problem

$$
u_{t t}-\Delta u+u+u_{t}+|u|^{2} u=u \ln |u|
$$

and investigated numerical solutions of this problem without theoretical analysis. Recently, Al-Gharabli et al. [7] considered the problem

$$
u_{t t}+\Delta^{2} u+u-\int_{0}^{t} h(t-s) \Delta^{2} u(s) d s=\alpha u \ln |u| \quad \text { in } \Omega \times(0, \infty)
$$

and proved existence and decay results of the solutions under the following condition on the relaxation function:

$$
h^{\prime}(t) \leq-\xi(t) h^{p}(t), \quad 1 \leq p<\frac{3}{2} .
$$

Al-Gharabli et al. [8] considered the problem

$$
\left|u_{t}\right|^{\rho} u_{t t}+\Delta^{2} u+\Delta^{2} u_{t t}-\int_{0}^{t} h(t-s) \Delta^{2} u(s) d s=\alpha u \ln |u| \quad \text { in } \Omega \times(0, \infty)
$$

and as in [7] proved the existence and decay results for the solutions with imposing the same condition (7). Very recently, Al-Gharabli [9] considered the same problem (6) and established a general decay result for which the relaxation function $h$ satisfies $h^{\prime}(t) \leq$ $-\xi(t) H(h(t))$. For more results on some problems with logarithmic nonlinearity, we refer to the recent works [10-14]. 


\subsection{Problems with infinite memory}

Giorgi et al. [15] considered the following semilinear hyperbolic equation with linear memory in a bounded domain $\Omega \subset \mathbb{R}^{3}$ :

$$
u_{t t}-K(0) \Delta u-\int_{0}^{+\infty} K^{\prime}(s) \Delta u(t-s) d s+g(u)=f \quad \text { in } \Omega \times \mathbb{R}_{+},
$$

with $K(0), K(+\infty)>0$ and $K^{\prime} \leq 0$ and proved the existence of global attractors for the solutions. Conti and Pata [16] considered the following semilinear hyperbolic equation:

$$
u_{t t}+\alpha u_{t}-K(0) \Delta u-\int_{0}^{+\infty} K^{\prime}(s) \Delta u(t-s) d s+g(u)=f \quad \text { in } \Omega \times \mathbb{R}_{+}
$$

where the memory kernel is a convex decreasing smooth function such that $K(0)>$ $K(+\infty)>0$, and $g: \mathbb{R}_{+} \rightarrow \mathbb{R}_{+}$is a nonlinear term of at most cubic growth satisfying some conditions. They proved the existence of a regular global attractor. Appleby et al. [17] studied the linear integro-differential equation

$$
u_{t t}+A u(t)+\int_{-\infty}^{t} K(t-s) A u(s) d s=0 \quad \text { for } t>0
$$

and established an exponential decay result for strong solutions in a Hilbert space. Pata [18] discussed the decay properties of the semigroup generated by the following equation:

$$
u_{t t}+\alpha A u(t)+\beta u_{t}(t)-\int_{0}^{+\infty} \mu(s) A u(t-s) d s=0 \quad \text { for } t>0,
$$

where $A$ is a strictly positive self-adjoint linear operator, $\alpha>0, \beta \geq 0$, and the memory kernel $\mu$ is a decreasing function satisfying specific conditions. Subsequently, they established necessary and sufficient conditions for the exponential stability. Guesmia [19] considered the equation

$$
u_{t t}+A u-\int_{0}^{+\infty} h(s) B u(t-s) d s=0 \quad \text { for } t>0
$$

and introduced a new ingenuous approach for proving a more general decay result based on the properties of convex functions and the generalized Young inequality. He used a larger class of infinite history kernels satisfying the condition

$$
\int_{0}^{+\infty} \frac{h(s)}{H^{-1}\left(-h^{\prime}(s)\right)} d s+\sup _{s \in \mathbb{R}_{+}} \frac{h(s)}{H^{-1}\left(-h^{\prime}(s)\right)}<+\infty
$$

with

$$
H(0)=H^{\prime}(0)=0 \text { and } \lim _{t \rightarrow+\infty} H^{\prime}(t)=+\infty,
$$

where $H: \mathbb{R}_{+} \rightarrow \mathbb{R}_{+}$is an increasing strictly convex function. Using this approach, Guesmia and Messaoudi [20] later considered the equation

$$
u_{t t}-\Delta u+\int_{0}^{t} h_{1}(t-s) \operatorname{div}\left(a_{1}(x) \nabla u(s)\right) d s+\int_{0}^{+\infty} h_{2}(s) \operatorname{div}\left(a_{2}(x) \nabla u(t-s)\right) d s=0
$$


in a bounded domain under suitable conditions on $a_{1}$ and $a_{2}$ for a wide class of relaxation functions $h_{1}$ and $h_{2}$, which are not necessarily decaying polynomially or exponentially, and established a general decay result such that the usual exponential and polynomial decay rates are only particular cases. Messaoudi and Al-Gharabli [7] considered the nonlinear wave equation

$$
\left|u_{t}\right|^{\rho} u_{t t}-\Delta u-\Delta u_{t t}+\int_{0}^{+\infty} h(s) \Delta u(t-s) d s=0 \quad \text { in } \Omega \times(0,+\infty),
$$

in which the relaxation function $g$ satisfies

$$
h^{\prime}(t) \leq-\xi(t) h(t), \quad t \geq 0,
$$

and they proved a general decay result on the solution energy using an approach different from that introduced by Guesmia [19]. Recently, Al-Mahdi and Al-Gharabli [21] considered the viscoelastic problem

$$
\left\{\begin{array}{l}
u_{t t}-\Delta u+\int_{0}^{+\infty} h(s) \Delta u(t-s) d s+\left|u_{t}\right|^{m-2} u_{t}=0 \quad \text { in } \Omega \times(0,+\infty), \\
u(x, t)=0 \quad \text { on } \partial \Omega \times(0,+\infty), \\
u(x,-t)=u_{0}(x, t), \quad u_{t}(x, 0)=u_{1}(x) \quad \text { in } \Omega \times(0,+\infty),
\end{array}\right.
$$

established decay results in which the relaxation function $h$ satisfies

$$
h^{\prime}(t) \leq-\xi(t) h^{p}(t), \quad t \geq 0,1 \leq p<\frac{3}{2},
$$

and obtained a better decay rate than that in [19] and [22]. For more results on problems with infinite memory and finite memory, we refer the reader to [23-27]. Motivated by all these works, we intend to establish a three-fold objective:

(a) To extend many earlier works for the wave equations such as those discussed in $[1,3,7,28-30]$ to the plate equation with logarithmic nonlinearity.

(b) To extend some general decay results, known for the case of finite history, to the case of infinite history where the relaxation function satisfies a wider class of relaxation functions instead of those considered in $[7,8,12,19,21,29,31]$.

(c) To drop the boundedness assumptions on the history data considered in many earlier results in $[7,19,21]$.

We obtain our results by using the multiplier method with some logarithmic inequalities and some properties of integro-differential equations and inequalities. Our decay result is based on $\xi, H$, and $\alpha$. This paper is organized as follows. In Sect. 2, we present some notations, assumptions, and a local and global existence result of our problem. In Sect. 3, we establish some lemmas needed in the proof of our result. Stability results with an example are presented in Sect. 4. Some conclusions are given in Sect. 5.

\section{Preliminaries}

In this section, we introduce our assumptions and give some useful lemmas. We use $c$ to denote a positive generic constant. 
(A1) $h: \mathbb{R}_{+} \rightarrow \mathbb{R}_{+}$is a $C^{1}$ nonincreasing function satisfying, for some $\beta_{0}>0$,

$$
-\beta_{0} h(s) \leq h^{\prime}(s), \quad h(t)>0 \quad \text { and } \quad 1-\int_{0}^{+\infty} h(s) d s:=\ell>0,
$$

(A2) $H:(0, \infty) \rightarrow(0, \infty)$ is a function in $C^{1}\left(\mathbb{R}_{+}\right) \cap C^{2}\left(\mathbb{R}_{+}^{*}\right)$ that is increasing and strictly convex, with $H(0)=H^{\prime}(0)=0$ and $\lim _{s \rightarrow+\infty} H^{\prime}(s)=+\infty, s \mapsto s H^{\prime}(s)$ and $s \mapsto$ $s\left(H^{\prime}\right)^{-1}(s)$ are convex on $(0, r]$, and there exists a nonincreasing function $\xi: \mathbb{R}_{+} \rightarrow$ $\mathbb{R}_{+}$such that

$$
h^{\prime}(t) \leq-\xi(t) H(h(t)), \quad t \geq 0 .
$$

(A3) The constant $\alpha$ in (1) is such that $0<\alpha<\alpha_{0}=\frac{2 \pi e^{3}}{c_{p}}$, where $c_{p}$ is the smallest positive number satisfying $\|\nabla u\|_{2}^{2} \leq c_{p}\|\Delta u\|_{2}^{2}$ for $u \in H_{0}^{2}(\Omega)$, where $\|\cdot\|_{2}=\|\cdot\|_{L^{2}(\Omega)}$.

Remark 2.1 Assumption (A3) is needed for establishing the local existence of the solutions of problem (1). For more details, we refer to [8].

Remark 2.2 If $H$ is a strictly increasing and strictly convex $C^{2}$ function on $(0, r]$ with $H(0)=H^{\prime}(0)=0$, then it has an extension $\bar{H}$ that is strictly increasing and strictly convex $C^{2}$ function on $(0,+\infty)$. For instance, if $H(r)=a, H^{\prime}(r)=b$, and $H^{\prime \prime}(r)=C$, we can define $\bar{H}$ for $t>r$ by

$$
\bar{H}(t)=\frac{C}{2} t^{2}+(b-C r) t+\left(a+\frac{C}{2} r^{2}-b r\right) .
$$

For simplicity, in the rest of this paper, we use $H$ instead of $\bar{H}$.

Remark 2.3 Since $H$ is strictly convex on $(0, r]$ and $H(0)=0$, then

$$
H(\theta t) \leq \theta H(t), \quad 0 \leq \theta \leq 1 \text { and } t \in(0, r] .
$$

Remark 2.4 The function $g(s)=\sqrt{\frac{2 \pi \ell}{c_{p} s}}-e^{-\frac{3}{2}}$ is a continuous decreasing function on $(0, \infty)$ with

$$
\lim _{s \rightarrow 0^{+}} g(s)=\infty \text { and } \quad \lim _{x \rightarrow \infty} g(x)=-e^{-\frac{3}{2}} .
$$

Then there exists a unique $\alpha_{0}>0$ such that $g\left(\alpha_{0}\right)=0$. Moreover,

$$
e^{-\frac{3}{2}}<\sqrt{\frac{2 \pi \ell}{c_{p} s}}, \quad s \in\left(0, \alpha_{0}\right),
$$

which implies that the selection of $\alpha$ in $(A 3)$ is possible.

The modified energy functional associated with problem (1)-(2) is given by

$$
\begin{aligned}
E(t)= & \frac{1}{\rho+2}\left\|u_{t}\right\|_{\rho+2}^{\rho+2}+\frac{\ell}{2}\|\Delta u\|_{2}^{2}+\frac{1}{2}\left\|\Delta u_{t}\right\|_{2}^{2}-\frac{\alpha}{2} \int_{\Omega} u^{2} \ln |u| d x \\
& +\frac{\alpha}{4}\|u\|_{2}^{2}+\frac{1}{2}(h \circ \Delta u),
\end{aligned}
$$


where

$$
(h \circ \Delta u)(t)=\int_{0}^{+\infty} h(s)\|\Delta u(s)-\Delta u(t-s)\|_{2}^{2} d s .
$$

Direct differentiation of (23) using (1)-(2) leads to

$$
E^{\prime}(t)=\frac{1}{2}\left(h^{\prime} \circ \Delta u\right)(t) \leq 0
$$

Lemma 2.1 ([32, 33] (Logarithmic Sobolev inequality)) Let $u$ be any function in $H_{0}^{1}(\Omega)$, and let a be any positive real number. Then

$$
\int_{\Omega} u^{2} \ln |u| d x \leq \frac{1}{2}\|u\|_{2}^{2} \ln \|u\|_{2}^{2}+\frac{a^{2}}{2 \pi}\|\nabla u\|_{2}^{2}-(1+\ln a)\|u\|_{2}^{2}
$$

Corollary 2.1 Let $u$ be any function in $H_{0}^{2}(\Omega)$, and let a be any positive real number. Then

$$
\int_{\Omega} u^{2} \ln |u| d x \leq \frac{1}{2}\|u\|_{2}^{2} \ln \|u\|_{2}^{2}+\frac{c_{p} a^{2}}{2 \pi}\|\Delta u\|_{2}^{2}-(1+\ln a)\|u\|_{2}^{2}
$$

Lemma 2.2 Let $\varepsilon_{0} \in(0,1)$. Then there exists $d_{\varepsilon_{0}}>0$ such that

$$
s|\ln s| \leq s^{2}+d_{\varepsilon_{0}} s^{1-\varepsilon_{0}}, \quad s>0 .
$$

Proof Let $f(s)=s^{\varepsilon_{0}}(|\ln s|-s)$. Note that $f$ is continuous on $(0, \infty)$, its limit at $0^{+}$is $0^{+}$, and its limit at $\infty$ is $-\infty$. Then $f$ has a maximum $d_{\varepsilon_{0}}$ on $(0, \infty)$, so (27) holds.

\subsection{Existence results}

In this subsection, we state without proof a local existence result of our problem (1)-(2).

Theorem 2.1 Let $\left(u_{0}, u_{1}\right) \in H_{0}^{2}(\Omega) \times H_{0}^{2}(\Omega)$. Assume that $(A 1)-(A 3)$ hold and

$$
e^{-\frac{3}{2}}<a<\sqrt{\frac{2 \pi \ell}{\alpha c_{p}}}
$$

Then problem (1)-(2) has a weak solution on $[0, T]$.

The proof of Theorem 2.1 can be obtained by following the same arguments as in [8] and adapting the finite history to the infinite case. For the global existence, we have the following:

Theorem 2.2 Assume that $(A 1)-(A 3)$ hold. Let $\left(u_{0}, u_{1}\right) \in H_{0}^{2}(\Omega) \times H_{0}^{2}(\Omega)$ be such that

$$
I(0)>0 \text { and } \sqrt{54} \alpha c_{*}^{3}\left(\frac{E(0)}{\ell}\right)^{\frac{1}{2}}<\ell,
$$

where $c_{*}^{3}$ is a positive embedding constant. Then we have: 
(i)

$$
I(t)>0, \quad t \in[0, T) .
$$

(ii) Problem (1)-(2) has a global weak solution,

where

$$
I(t):=\ell\|\Delta u\|_{2}^{2}+\left\|\Delta u_{t}\right\|_{2}^{2}+(h \circ \Delta u)(t)-3 \alpha \int_{\Omega} u^{2} \ln |u| d x
$$

and

$$
J(t):=\frac{1}{3}\left[\ell\|\Delta u\|_{2}^{2}+\left\|\Delta u_{t}\right\|_{2}^{2}+g \circ \Delta u\right]+\frac{k}{4}\|u\|_{2}^{2}+\frac{1}{6} I(t) .
$$

The proof of Theorem 2.2 can be obtained by following the same arguments as in [8] by adapting the finite memory to infinite memory.

\section{Technical lemmas}

In this section, we start by establishing several lemmas needed for the proof of our main result.

Lemma 3.1 There exists a positive constant $M_{1}$ such that

$$
\int_{t}^{\infty} h(s)(\Delta u(t)-\Delta u(t-s))^{2} d s d x \leq M_{1} h_{1}(t)
$$

where $h_{1}(t):=\int_{0}^{+\infty} h(t+s)\left(1+\left\|\Delta u_{0}(s)\right\|^{2}\right) d s$.

Proof The proof is based on some arguments in [30]. In fact, we have

$$
\begin{aligned}
\int_{t}^{+\infty} & h(s)\|\Delta u(t)-\Delta u(t-s)\|^{2} d s \\
& \leq 2\|\Delta u(t)\|^{2} \int_{t}^{+\infty} h(s) d s+2 \int_{t}^{+\infty} h(s)\|\Delta u(t-s)\|^{2} d s \\
& \leq 2 \sup _{s \geq 0}\|\Delta u(s)\|^{2} \int_{0}^{+\infty} h(t+s) d s+2 \int_{0}^{+\infty} g(t+s)\|\Delta u(-s)\|^{2} d s \\
& \leq\left(\frac{4}{\ell} E(s)\right) \int_{0}^{\infty} h(t+s) d s+2 \int_{0}^{\infty} h(t+s)\left\|\Delta u_{0}(s)\right\|^{2} d s \\
& \leq\left(\frac{4}{\ell} E(0)\right) \int_{0}^{+\infty} h(t+s) d s+2 \int_{0}^{+\infty} h(t+s)\left\|\Delta u_{0}(s)\right\|^{2} d s \\
& \leq M_{1} \int_{0}^{+\infty} h(t+s)\left(1+\left\|\Delta u_{0}(s)\right\|^{2}\right) d s,
\end{aligned}
$$

where $M_{1}=\max \left\{2, \frac{4 E(0)}{\ell}\right\}$. 
Lemma 3.2 Assume that hatisfies (A1). Then, for $u \in H_{0}^{2}(\Omega)$,

$$
\begin{aligned}
& \int_{\Omega}\left(\int_{0}^{+\infty} h(s)(u(t)-u(t-s)) d s\right)^{2} d x \leq c(h \circ \Delta u)(t), \\
& \int_{\Omega}\left(\int_{0}^{+\infty} h^{\prime}(s)(u(t)-u(t-s)) d s\right)^{2} d x \leq-c\left(h^{\prime} \circ \Delta u\right)(t) .
\end{aligned}
$$

Proof The proof can be easily obtained by applying the Cauchy-Schwarz and Poincaré inequalities.

Lemma 3.3 Assume that (A1)-(A3) and (29) hold. Then the functionals

$$
\begin{aligned}
& \psi(t):=\frac{1}{\rho+1} \int_{\Omega}\left|u_{t}\right|^{\rho} u_{t} u d x+\int_{\Omega} \Delta u \Delta u_{t} d x, \\
& \chi(t):=-\int_{\Omega}\left(\Delta^{2} u_{t}+\frac{1}{\rho+1}\left|u_{t}\right|^{\rho} u_{t}\right) \int_{0}^{+\infty} h(s)(u(t)-u(t-s)) d s d x,
\end{aligned}
$$

satisfy, along the solutions of (1)-(2), the following estimates for any $\delta, \delta_{1}, \delta_{2}>0$ and $\varepsilon_{0} \in$ $(0,1)$ :

$$
\begin{aligned}
\psi^{\prime}(t) \leq & -\frac{\ell}{2} \int_{\Omega}|\Delta u|^{2} d x+\int_{\Omega}\left|\Delta u_{t}\right|^{2} d x+\frac{1}{\rho+1} \int_{\Omega}\left|u_{t}\right|^{\rho+2} d x+c(h \circ \Delta u)(t) \\
& +\alpha \int_{\Omega} u^{2} \ln |u| d x, \\
\chi^{\prime}(t) \leq & {\left[\left(1+2(1-\ell)^{2}\right) \delta_{1}+\frac{\delta}{4}\right] \int_{\Omega}|\Delta u|^{2} d x-\frac{(1-\ell)}{\rho+1} \int_{\Omega}\left|u_{t}\right|^{\rho+2} d x } \\
& +c\left(\delta_{1}+\frac{1}{\delta_{1}}+\frac{1}{\delta}\right)(h \circ \Delta u)(t)-\frac{c}{\delta_{2}}\left(h^{\prime} \circ \nabla u\right)(t) \\
& +\left[\delta_{2}+c \delta_{2}(E(0))^{\rho}-(1-\ell)\right] \int_{\Omega}\left|\Delta u_{t}\right|^{2} d x+c_{\varepsilon_{0}, \delta}(h \circ \Delta u)^{\frac{1}{1+\varepsilon_{0}}}(t) .
\end{aligned}
$$

Proof The proof of Lemma 3.3 is similar to that in [8] with some adjustments according to the infinite memory case.

Lemma 3.4 Assume that (A1)-(A3) and (29) hold and let $\varepsilon_{0} \in(0,1)$. Assume that

$$
0<E(0)<\frac{e \ell \pi}{4 c_{p}} .
$$

Then, for $\alpha$ small enough, there exist positive constants $\varepsilon$ and $N$ such that the functional

$$
L:=N E+\varepsilon \psi+\chi
$$

satisfies

$$
L \sim E,
$$


and, for any $t \geq 0$, there exists a positive constant $m$ such that

$$
L^{\prime}(t) \leq-m E(t)+c(h \circ \Delta u)(t)+c_{\varepsilon_{0}}(h \circ \Delta u)^{\frac{1}{1+\varepsilon_{0}}}(t) .
$$

Proof For the proof of (38), we refer to [8]. To prove (39), we let $\int_{0}^{+\infty} h(s) d s=: h_{0}$ and using (24), (35), and (36), for $t \geq 0$, we have

$$
\begin{aligned}
L^{\prime}(t) \leq & \left(\frac{N}{2}-\frac{c}{\delta_{2}}\right)\left(h^{\prime} \circ \Delta u\right)(t)-\frac{h_{0}-\varepsilon}{\rho+1} \int_{\Omega}\left|u_{t}\right|^{\rho+2} d x \\
& -\left[\varepsilon \frac{\ell}{2}-\left(1+2(1-\ell)^{2}\right) \delta_{1}-\frac{\delta}{4}\right]\|\Delta u\|_{2}^{2} \\
& -\left[h_{0}-\varepsilon-\delta_{2}-c \delta_{2}(E(0))^{\rho}\right]\left\|\Delta u_{t}\right\|_{2}^{2} \\
& +c\left(\varepsilon+\delta_{1}+\frac{1}{\delta_{1}}+\frac{1}{\delta}\right)(h \circ \Delta u)(t) \\
& +c_{\varepsilon_{0}, \delta}(h \circ \Delta u)^{\frac{1}{1+\varepsilon_{0}}}(t)+\varepsilon \alpha \int_{\Omega} u^{2} \ln |u| d x .
\end{aligned}
$$

Using the definition of $E(t)$, we obtain, for any $m>0$,

$$
\begin{aligned}
L^{\prime}(t) \leq & -m E(t)+\left(\frac{N}{2}-\frac{c}{\delta_{2}}\right)\left(h^{\prime} \circ \Delta u\right)(t)-\left(\frac{h_{0}-\varepsilon}{\rho+1}-\frac{m}{\rho+2}\right) \int_{\Omega}\left|u_{t}\right|^{\rho+2} d x \\
& -\left[\varepsilon \frac{\ell}{2}-\left(1+2(1-\ell)^{2}\right) \delta_{1}-\frac{\delta}{4}-\frac{m\left(1-h_{0}\right)}{2}\right]\|\Delta u\|_{2}^{2} \\
& -\left[h_{0}-\varepsilon-\delta_{2}-c \delta_{2}(E(0))^{\rho}-\frac{m}{2}\right]\left\|\Delta u_{t}\right\|_{2}^{2} \\
& +\left[c\left(\varepsilon+\delta_{1}+\frac{1}{\delta_{1}}+\frac{1}{\delta}\right)+\frac{m}{2}\right](h \circ \Delta u)(t) \\
& +c_{\varepsilon_{0}, \delta}(h \circ \Delta u)^{\frac{1}{1+\varepsilon_{0}}}(t)+\frac{m \alpha}{4}\|u\|_{2}^{2} \\
& +\left(\varepsilon-\frac{m}{2}\right) \alpha \int_{\Omega} u^{2} \ln |u| d x .
\end{aligned}
$$

Using the logarithmic Sobolev inequality (26), we get, for $0<m<2 \varepsilon$,

$$
\begin{aligned}
L^{\prime}(t) \leq & -m E(t)+\left[\frac{N}{2}-\frac{c}{\delta_{2}}\right]\left(h^{\prime} \circ \Delta u\right)(t)-\left(\frac{h_{0}-\varepsilon}{\rho+1}-\frac{m}{\rho+2}\right) \int_{\Omega}\left|u_{t}\right|^{\rho+2} d x \\
& -\left[\varepsilon \frac{\ell}{2}-\left(1+2(1-\ell)^{2}\right) \delta_{1}-\frac{\delta}{4}-\frac{m\left(1-h_{0}\right)}{2}-\left(\varepsilon-\frac{m}{2}\right) \frac{\alpha c_{p} a^{2}}{2 \pi}\right]\|\Delta u\|_{2}^{2} \\
& -\left(h_{0}-\varepsilon-\delta_{2}-c \delta_{2}(E(0))^{\rho}-\frac{m}{2}\right)\left\|\Delta u_{t}\right\|_{2}^{2} \\
& +\left[c\left(\varepsilon+\delta_{1}+\frac{1}{\delta_{1}}+\frac{1}{\delta}\right)+\frac{m}{2}\right](h \circ \Delta u)(t)+c_{\varepsilon_{0}, \delta}(h \circ \Delta u)^{\frac{1}{1+\varepsilon_{0}}}(t) \\
& -\left(\varepsilon-\frac{m}{2}\right) \frac{\alpha}{2}\left(2(1+\ln a)-\ln \|u\|_{2}^{2}\right)\|u\|_{2}^{2}+\frac{m \alpha}{4}\|u\|_{2}^{2} .
\end{aligned}
$$


At this point, we carefully choose our constant. First, we pick $0<\varepsilon<h_{0}$. Then for $\delta_{1}, \delta_{2}$, and $\delta$ small enough, we have

$$
k_{1}:=\varepsilon \frac{\ell}{2}-\left(1+2(1-\ell)^{2}\right) \delta_{1}-\frac{\delta}{4}>0
$$

and

$$
k_{2}:=h_{0}-\varepsilon-\delta_{2}-c \delta_{2}(E(0))^{\rho}>0
$$

Then, for $N$ sufficiently large,

$$
N>c(1+\varepsilon) \text { and } \quad \frac{N}{2}-\frac{c}{\delta_{2}} \geq 0 .
$$

Consequently, we get

$$
\begin{aligned}
L^{\prime}(t) \leq & -m E(t)-\left(\frac{h_{0}-\varepsilon}{\rho+1}-\frac{m}{\rho+2}\right) \int_{\Omega}\left|u_{t}\right|^{\rho+2} d x \\
& -\left[k_{1}-\frac{m\left(1-h_{0}\right)}{2}-\left(\varepsilon-\frac{m}{2}\right) \frac{\alpha c_{p} a^{2}}{2 \pi}\right]\|\Delta u\|_{2}^{2} \\
& -\left(k_{2}-\frac{m}{2}\right)\left\|\Delta u_{t}\right\|_{2}^{2}+\left(c+\frac{m}{2}\right)(h \circ \Delta u)(t) \\
& +c_{\varepsilon_{0}}(h \circ \Delta u)^{\frac{1}{1+\varepsilon_{0}}}(t)+\frac{m \alpha}{4}\|u\|_{2}^{2} \\
& -\left(\varepsilon-\frac{m}{2}\right) \frac{\alpha}{2}\left(2(1+\ln a)-\ln \|u\|_{2}^{2}\right)\|u\|_{2}^{2} .
\end{aligned}
$$

Finally, we choose $m$ and $\alpha$ small enough so that $m \leq \varepsilon$ (so $\left.\frac{m \alpha}{4} \leq\left(\varepsilon-\frac{m}{2}\right) \frac{\alpha}{2}\right)$,

$$
\begin{aligned}
& \frac{h_{0}-\varepsilon}{\rho+1}-\frac{m}{\rho+2}>0, \\
& k_{1}-\frac{m\left(1-h_{0}\right)}{2}-\left(\varepsilon-\frac{m}{2}\right) \frac{\alpha c_{p} a^{2}}{2 \pi}>0,
\end{aligned}
$$

and

$$
k_{2}-\frac{m}{2}>0
$$

and we get

$$
\begin{aligned}
L^{\prime}(t) \leq & -m E(t)+c(h \circ \Delta u)(t)+c_{\varepsilon_{0}}(h \circ \Delta u)^{\frac{1}{1+\varepsilon_{0}}}(t) \\
& -\left(\varepsilon-\frac{m}{2}\right) \frac{\alpha}{2}\left(1+2 \ln a-\ln \|u\|_{2}^{2}\right)\|u\|_{2}^{2} .
\end{aligned}
$$

Using (23), (24), (30), (31), (32), and (37), we have

$$
\ln \|u\|_{2}^{2} \leq \ln \left(\frac{4}{\alpha} J(t)\right) \leq \ln \left(\frac{4}{\alpha} E(t)\right) \leq \ln \left(\frac{4}{\alpha} E(0)\right) \leq \ln \left(\frac{e \ell \pi}{\alpha c_{p}}\right) .
$$


By choosing $a$ satisfying

$$
\max \left\{e^{-\frac{3}{2}}, \sqrt{\frac{\ell \pi}{\alpha c_{p}}}\right\}<a<\sqrt{\frac{2 \ell \pi}{\alpha c_{p}}}
$$

we achieve that (28) is satisfied. This selection gives a guarantee that

$$
1+2 \ln a-\ln \|u\|_{2}^{2} \geq 0,
$$

which completes the proof of (39).

Remark 3.1 Recalling (23), (24), (30), and (32), we have

$$
E(0) \geq E(t)=J(t)+\frac{1}{\rho+2}\left\|u_{t}\right\|_{\rho+2}^{\rho+2} \geq J(t) \geq \frac{1}{3}(h \circ \Delta u)(t),
$$

which gives

$$
(h \circ \Delta u)(t) \leq 3 E(0) .
$$

Using (47), for any $\varepsilon_{0} \in(0,1)$, we obtain that

$$
\begin{aligned}
(h \circ \Delta u)(t) & =(h \circ \Delta u)^{\frac{\varepsilon_{0}}{1+\varepsilon_{0}}}(t)(h \circ \Delta u)^{\frac{1}{1+\varepsilon_{0}}}(t) \\
& \leq c(h \circ \Delta u)^{\frac{1}{1+\varepsilon_{0}}}(t) .
\end{aligned}
$$

Lemma 3.5 If $(A 1)-(A 2)$ are satisfied, then we have, for all $t>0$, the estimate

$$
\int_{0}^{t} h(s)\|\Delta u(t)-\Delta u(t-s)\|_{2}^{2} d s \leq \frac{t+1}{q_{0}} H^{-1}\left(\frac{q_{0} \mu(t+1)}{t \xi(t)}\right),
$$

where $q_{0}>0$ is small enough, $H$ is defined in Remark 2.2, and

$$
\mu(t):=-\int_{0}^{t} h^{\prime}(s)\|\Delta u(t)-\Delta u(t-s)\|_{2}^{2} d s \leq-c E^{\prime}(t) .
$$

Proof To establish (49), we introduce the functional

$$
\lambda(t):=\frac{q_{0}}{t+1} \int_{0}^{t}\|\Delta u(t)-\Delta u(t-s)\|_{2}^{2} d s .
$$


Then since $E$ is nonincreasing, by (23) we get

$$
\begin{aligned}
\lambda(t) & \leq \frac{2 q_{0}}{t+1}\left(\int_{0}^{t}\|\Delta u(t)\|_{2}^{2}+\int_{0}^{t}\|\Delta u(t-s)\|_{2}^{2} d s\right) \\
& \leq \frac{4 q_{0}}{\ell(t+1)}\left(\int_{0}^{t}(E(t)+E(t-s)) d s\right) \\
& \leq \frac{8 q_{0}}{\ell(t+1)} \int_{0}^{t} E(s) d s \\
& \leq \frac{8 q_{0}}{\ell(t+1)} \int_{0}^{t} E(0) d s \\
& <+\infty .
\end{aligned}
$$

Thus $q_{0}$ can be chosen so small so that, for all $t>0$,

$$
\lambda(t)<1
$$

Without loss of generality, for all $t>0$, we assume that $\lambda(t)>0$; otherwise, we get an exponential decay from (39). Using Jensen's inequality, (2.3), (50), and (53) gives

$$
\begin{aligned}
\mu(t) & =\frac{1}{q_{0} \lambda(t)} \int_{0}^{t} \lambda(t)\left(-h^{\prime}(s)\right) \int_{\Omega} q_{0}|\Delta u(t)-\Delta u(t-s)|^{2} d x d s \\
& \geq \frac{1}{q_{0} \lambda(t)} \int_{0}^{t} \lambda(t) \xi(s) H(h(s)) \int_{\Omega} q_{0}|\Delta u(t)-\Delta u(t-s)|^{2} d x d s \\
& \geq \frac{\xi(t)}{q_{0} \lambda(t)} \int_{0}^{t} H(\lambda(t) h(s)) \int_{\Omega} q_{0}|\Delta u(t)-\Delta u(t-s)|^{2} d x d s \\
& \geq \frac{(t+1) \xi(t)}{q_{0}} H\left(\frac{q_{0}}{(t+1)} \int_{0}^{t} h(s) \int_{\Omega}|\Delta u(t)-\Delta u(t-s)|^{2} d x d s\right) \\
& =\frac{(t+1) \xi(t)}{q_{0}} H\left(\frac{q_{0}}{(t+1)} \int_{0}^{t} h(s) \int_{\Omega}|\Delta u(t)-\Delta u(t-s)|^{2} d x d s\right),
\end{aligned}
$$

and hence (49) is established.

\section{Decay result}

In this section, we state and prove our main result and provide an example to illustrate our decay results. Let us start introducing some functions and then establishing several lemmas needed for the proof of our main result. As in [30], we introduce the following functions:

$$
\begin{aligned}
& G_{1}(t):=\int_{t}^{1} \frac{1}{s G^{\prime}(s)} d s, \\
& G_{2}(t)=t G^{\prime}(t), \quad G_{3}(t)=t\left(G^{\prime}\right)^{-1}(t), \quad G_{4}(t)=G_{3}^{*}(t),
\end{aligned}
$$

where $G^{-1}(t)=\left(H^{-1}(t)\right)^{\frac{1}{1+\varepsilon_{0}}}$ and $\varepsilon_{0} \in(0,1)$. Further, we introduce the class $S$ of functions $\chi: \mathbb{R}_{+} \rightarrow \mathbb{R}_{+}^{*}$ satisfying, for fixed $c_{1}, c_{2}>0$ (should be selected carefully in (76)),

$$
\chi \in C^{1}\left(\mathbb{R}_{+}\right), \quad \chi \leq 1, \chi^{\prime} \leq 0
$$


and

$$
c_{2} G_{4}\left[\frac{c}{d} q(t) h_{0}(t)\right] \leq c_{1}\left(G_{2}\left(\frac{G_{5}(s)}{\chi(s)}\right)-\frac{G_{2}\left(G_{5}(t)\right)}{\chi(t)}\right),
$$

where $d>0, c$ is a generic positive constant that may change from line to line, $h_{2}$ and $q$ will be defined later in the proof of our main result, and

$$
G_{5}(t)=G_{1}^{-1}\left(c_{1} \int_{0}^{t} \xi(s) d s\right)
$$

Remark 4.1 According to the properties of $H$ introduced in $(A 2)$ and the definition of $G$, we can see that $G^{\prime}>0$ and $G^{\prime \prime}>0$ on $(0, r], G_{2}$ is convex increasing and defines a bijection from $\mathbb{R}_{+}$to $\mathbb{R}_{+}, G_{1}$ is decreasing and defines a bijection from $(0,1]$ to $\mathbb{R}_{+}$, and $G_{3}$ and $G_{4}$ are convex increasing functions on $(0, r]$. Then the set $S$ is not empty because it contains $\chi(s)=\varepsilon G_{5}(s)$ with $0<\varepsilon \leq 1$ small enough. Indeed, (57) is satisfied (since (55) and (59)).

Theorem 4.1 Assume that (A1)-(A3) and (29) hold. Then for any $\chi$ satisfying (57) and (58) and for any $\varepsilon_{0} \in(0,1)$, there exists a strictly positive constant $C$ such that the solution of (1)-(2) satisfies, for all $t \geq 0$,

$$
E(t) \leq \frac{C G_{5}(t)}{\chi(t) q(t)}
$$

where $G_{5}$ and $\chi$ are defined in (55) and (57), respectively, and $q$ will be defined later in the proof.

Proof Using (39), (48), and (49), for some positive constant $m, \varepsilon_{0} \in(0,1)$, and any $t \geq 0$, we get

$$
L^{\prime}(t) \leq-m E(t)+c\left(\frac{t+1}{q_{0}}\right)^{\frac{1}{1+\varepsilon_{0}}}\left(H^{-1}\left(\frac{q_{0} \mu(t)}{(t+1) \xi(t)}\right)\right)^{\frac{1}{1+\varepsilon_{0}}}(t)+c h_{1}^{\frac{1}{1+\varepsilon_{0}}}(t) .
$$

Combining the strict increasing of $H$ and the inequality $\frac{1}{t+1}<1$ for $t>0$, we obtain

$$
H^{-1}\left(\frac{q_{0} \mu(t)}{(t+1) \xi(t)}\right) \leq H^{-1}\left(\frac{q_{0} \mu(t)}{(t+1)^{\frac{1}{1+\varepsilon_{0}} \xi(t)}}\right)
$$

and, then (61) becomes, for any $t \geq 0$ and $\varepsilon_{0} \in(0,1)$,

$$
L^{\prime}(t) \leq-m E(t)+c_{\varepsilon_{0}} \frac{(t+1)^{\frac{1}{1+\varepsilon_{0}}}}{q_{0}}\left(H^{-1}\left(\frac{q_{0} \mu(t)}{(t+1)^{\frac{1}{1+\varepsilon_{0}}} \xi(t)}\right)\right)^{\frac{1}{1+\varepsilon_{0}}}(t)+\operatorname{ch}_{1}^{\frac{1}{1+\varepsilon_{0}}}(t)
$$

For simplicity, we let $q(t):=q_{0}(t+1)^{\frac{-1}{1+\varepsilon_{0}}}$ and $h_{2}(t):=c h_{1}^{\frac{1}{1+\varepsilon_{0}}}(t)$. Then (63) becomes

$$
L^{\prime}(t) \leq-m E(t)+\frac{c_{\varepsilon_{0}}}{\gamma(t)}\left(H^{-1}\left(\frac{q(t) \mu(t)}{\xi(t)}\right)\right)^{\frac{1}{1+\varepsilon_{0}}}(t)+c h_{2}(t)
$$


Further, letting $G^{-1}(t)=\left(H^{-1}(t)\right)^{\frac{1}{1+\varepsilon_{0}}}$ we reduce (64) to

$$
L^{\prime}(t) \leq-m E(t)+\frac{c_{\varepsilon_{0}}}{\gamma(t)} G^{-1}\left(\frac{q(t) \mu(t)}{\xi(t)}\right)+c h_{2}(t), \quad t \geq 0
$$

For $\varepsilon_{1}<r$, let the functional $\mathcal{F}$ be defined by

$$
\mathcal{F}(t):=G^{\prime}\left(\varepsilon_{1} \frac{E(t) q(t)}{E(0)}\right) L(t),
$$

which satisfies $\mathcal{F} \sim E$. Noting that $G^{\prime \prime} \geq 0, q^{\prime} \leq 0$, and $E^{\prime} \leq 0$, we get

$$
\begin{aligned}
\mathcal{F}^{\prime}(t)= & \varepsilon_{1} \frac{(q E)^{\prime}(t)}{E(0)} G^{\prime \prime}\left(\varepsilon_{1} \frac{E(t) q(t)}{E(0)}\right) L(t)+G^{\prime}\left(\varepsilon_{1} \frac{E(t) q(t)}{E(0)}\right) L^{\prime}(t) \\
\leq & -m E(t) G^{\prime}\left(\varepsilon_{1} \frac{E(t) q(t)}{E(0)}\right)+\frac{c}{q(t)} G^{\prime}\left(\varepsilon_{1} \frac{E(t) q(t)}{E(0)}\right) G^{-1}\left(\frac{q(t) \mu(t)}{\xi(t)}\right) \\
& +c_{2}(t) G^{\prime}\left(\varepsilon_{1} \frac{E(t) q(t)}{E(0)}\right) .
\end{aligned}
$$

Let $G^{*}$ be the convex conjugate of $G$ in the sense of Young (see [34]). Then

$$
G^{*}(s)=s\left(G^{\prime}\right)^{-1}(s)-G\left[\left(G^{\prime}\right)^{-1}(s)\right] \text { for } s \in\left(0, G^{\prime}(r)\right],
$$

and $G^{*}$ satisfies the generalized Young inequality

$$
A B \leq G^{*}(A)+G(B) \quad \text { if } A \in\left(0, G^{\prime}(r)\right], B \in(0, r] .
$$

So, with $A=G^{\prime}\left(\varepsilon_{1} \frac{E(t) q(t)}{E(0)}\right)$ and $B=G^{-1}\left(\frac{q(t) \mu(t)}{\xi(t)}\right)$, using (24) and (66)-(68), we arrive at

$$
\begin{aligned}
\mathcal{F}^{\prime}(t) \leq & -m E(t) G^{\prime}\left(\varepsilon_{1} \frac{E(t) q(t)}{E(0)}\right)+\frac{c}{q(t)} G^{*}\left(G^{\prime}\left(\varepsilon_{1} \frac{E(t) q(t)}{E(0)}\right)\right)+c\left(\frac{\mu(t) q(t)}{\xi(t)}\right) \\
& +c h_{2}(t) G^{\prime}\left(\varepsilon_{1} \frac{E(t) q(t)}{E(0)}\right) \\
\leq & -m E(t) G^{\prime}\left(\varepsilon_{1} \frac{E(t) q(t)}{E(0)}\right)+c \varepsilon_{1} \frac{E(t)}{E(0)} G^{\prime}\left(\varepsilon_{1} \frac{E(t) q(t)}{E(0)}\right)+c\left(\frac{\mu(t) q(t)}{\xi(t)}\right) \\
& +c h_{2}(t) G^{\prime}\left(\varepsilon_{1} \frac{E(t) q(t)}{E(0)}\right) .
\end{aligned}
$$

Multiplying (69) by $\xi(t)$, using (50), and the facts that $\varepsilon_{1} \frac{E(t) q(t)}{E(0)}<r$ and $G^{\prime}\left(\varepsilon_{1} \frac{E(t) q(t)}{E(0)}\right)=$ $G^{\prime}\left(\varepsilon_{1} \frac{E(t) q(t)}{E(0)}\right)$, we get

$$
\begin{aligned}
\xi(t) \mathcal{F}^{\prime}(t) \leq & -m \xi(t) E(t) G^{\prime}\left(\varepsilon_{1} \frac{E(t) q(t)}{E(0)}\right)+c \xi(t) \varepsilon_{1} \frac{E(t)}{E(0)} G^{\prime}\left(\varepsilon_{1} \frac{E(t) q(t)}{E(0)}\right) \\
& +c \mu(t) q(t)+c \xi(t) h_{2}(t) G^{\prime}\left(\varepsilon_{1} \frac{E(t) q(t)}{E(0)}\right) \\
\leq & -\left(\frac{m E(0)}{\varepsilon_{1}}-c\right) \xi(t) \varepsilon_{1} \frac{E(t)}{E(0)} G^{\prime}\left(\varepsilon_{1} \frac{E(t) q(t)}{E(0)}\right) \\
& -c E^{\prime}(t)+c \xi(t) h_{2}(t) G^{\prime}\left(\varepsilon_{1} \frac{E(t) q(t)}{E(0)}\right) .
\end{aligned}
$$


Consequently, recalling the definition of $G_{2}$ and choosing $\varepsilon_{1}$ such that $k=\left(\frac{m E(0)}{\varepsilon_{1}}-c\right)>0$, we obtain, for all $t \in \mathbb{R}_{+}$,

$$
\begin{aligned}
\mathcal{F}_{1}^{\prime}(t) & \leq-k \varepsilon_{1} \xi(t)\left(\frac{E(t)}{E(0)}\right) G^{\prime}\left(\varepsilon_{1} \frac{E(t) q(t)}{E(0)}\right)+c \xi(t) h_{2}(t) G^{\prime}\left(\varepsilon_{1} \frac{E(t) q(t)}{E(0)}\right) \\
& =-k \frac{\xi(t)}{q(t)} G_{2}\left(\frac{E(t) q(t)}{E(0)}\right)+c \xi(t) h_{2}(t) G^{\prime}\left(\varepsilon_{1} \frac{E(t) q(t)}{E(0)}\right)
\end{aligned}
$$

where $\mathcal{F}_{1}=\xi \mathcal{F}+c E \sim E$ satisfies, for some $\alpha_{1}, \alpha_{2}>0$,

$$
\alpha_{1} \mathcal{F}_{1}(t) \leq E(t) \leq \alpha_{2} \mathcal{F}_{1}(t)
$$

Since $G_{2}^{\prime}(t)=G^{\prime}(t)+t G^{\prime \prime}(t)$, using the strict convexity of $G$ on $(0, r]$, we find that $G_{2}^{\prime}(t), G_{2}(t)>0$ on $(0, r]$. Applying the general Young inequality (68) to the last term in (71) with $A=G^{\prime}\left(\varepsilon_{1} \frac{E(t) q(t)}{E(0)}\right)$ and $B=\left[\frac{c}{d} h_{2}(t)\right]$, we have

$$
\begin{aligned}
c h_{2}(t) G^{\prime}\left(\varepsilon_{1} \frac{E(t) q(t)}{E(0)}\right) & =\frac{d}{q(t)}\left[\frac{c}{d} q(t) h_{2}(t)\right]\left(G^{\prime}\left(\varepsilon_{1} \frac{E(t) q(t)}{E(0)}\right)\right) \\
& \leq \frac{d}{q(t)} G_{3}\left(G^{\prime}\left(\varepsilon_{1} \frac{E(t) q(t)}{E(0)}\right)\right)+\frac{d}{q(t)} G_{3}^{*}\left[\frac{c}{d} q(t) h_{2}(t)\right] \\
& \leq \frac{d}{q(t)}\left(\varepsilon_{1} \frac{E(t) q(t)}{E(0)}\right)\left(G^{\prime}\left(\varepsilon_{1} \frac{E(t) q(t)}{E(0)}\right)\right)+\frac{d}{q(t)} G_{4}\left[\frac{c}{d} q(t) h_{2}(t)\right] \\
& \leq \frac{d}{q(t)} G_{2}\left(\varepsilon_{1} \frac{E(t) q(t)}{E(0)}\right)+\frac{d}{q(t)} G_{4}\left[\frac{c}{d} q(t) h_{2}(t)\right] .
\end{aligned}
$$

Now, combining (71) and (73) and choosing $d$ small enough so that $k_{1}=(k-d)>0$, we arrive at

$$
\begin{aligned}
\mathcal{F}_{1}^{\prime}(t) & \leq-k \frac{\xi(t)}{q(t)} G_{2}\left(\varepsilon_{1} \frac{E(t) q(t)}{E(0)}\right)+\frac{d \xi(t)}{q(t)} G_{2}\left(\varepsilon_{1} \frac{E(t) q(t)}{E(0)}\right)+\frac{d \xi(t)}{q(t)} G_{4}\left[\frac{c}{d} q(t) h_{2}(t)\right] \\
& \leq-k_{1} \frac{\xi(t)}{q(t)} G_{2}\left(\varepsilon_{1} \frac{E(t) q(t)}{E(0)}\right)+\frac{d \xi(t)}{q(t)} G_{4}\left[\frac{c}{d} q(t) h_{2}(t)\right] .
\end{aligned}
$$

Using the equivalent property in (72) and the nonincrease of $G_{2}$, we have, for some $d_{0}=$ $\frac{\alpha_{1}}{E(0)}>0$,

$$
G_{2}\left(\varepsilon_{1} \frac{E(t) q(t)}{E(0)}\right) \geq G_{2}\left(d_{0} \mathcal{F}_{1}(t) q(t)\right)
$$

Letting $\mathcal{F}_{2}(t):=d_{0} \mathcal{F}_{1}(t) q(t)$ and recalling that $q^{\prime} \leq 0$, we arrive at,

$$
\mathcal{F}_{2}^{\prime}(t) \leq d_{0} q(t)\left(-k_{1} \frac{\xi(t)}{q(t)} \Psi_{2}\left(\varepsilon_{0} \frac{E(t) q(t)}{E(0)}\right)+\frac{d \xi(t)}{q(t)} \Psi_{4}\left[\frac{c}{d} q(t) h_{0}(t)\right]\right) .
$$

Then (75) becomes, for some constants $c_{1}=d_{0} k_{1}>0$ and $c_{2}=d_{0} d>0$,

$$
\mathcal{F}_{2}^{\prime}(t) \leq-c_{1} \xi(t) G_{2}\left(\mathcal{F}_{2}(t)\right)+c_{2} \xi(t) G_{4}\left[\frac{c}{d} q(t) h_{2}(t)\right] .
$$


Since $d_{0} q(t)$ is nonincreasing. Using the equivalent property $\mathcal{F}_{1} \sim E$ implies that there exists $b_{0}>0$ such that $\mathcal{F}_{2}(t) \geq b_{0} E(t) q(t)$. Since $\chi(t)$ satisfies (57) and (58), if $b_{0} q(t) E(t) \leq$ $2 \frac{G_{5}(t)}{\chi(t)}$, then we get

$$
E(t) \leq \frac{2}{b_{0}} \frac{G_{5}(t)}{\chi(t) q(t)}
$$

If $b_{0} q(t) E(t)>2 \frac{G_{5}(t)}{\chi(t)}$, then since $q(t) E(t)$ is a nonincreasing function, for any $0 \leq s \leq t$, we have $b_{0} q(s) E(s)>2 \frac{G_{5}(t)}{\chi(t)}$. Therefore, for any $0 \leq s \leq t$,

$$
\mathcal{F}_{2}(s)>2 \frac{G_{5}(t)}{\chi(t)}
$$

Using (21), $0<\chi \leq 1$, and the convexity of $G_{2}$, we have, for any $0<\varepsilon_{2} \leq 1$,

$$
\begin{aligned}
G_{2}\left(\varepsilon_{2} \chi(s) \mathcal{F}_{2}(s)-\varepsilon_{2} G_{5}(s)\right) & =G_{2}\left(\varepsilon_{2} \chi(s) \mathcal{F}_{2}(s)-\frac{\varepsilon_{2} \chi(s) G_{5}(s)}{\chi(s)}\right) \\
& \leq \varepsilon_{2} \chi(s) G_{2}\left(\mathcal{F}_{2}(s)-\frac{G_{5}(s)}{\chi(s)}\right) .
\end{aligned}
$$

Recalling the definition of $G_{2}$, that is, $G_{2}(t)=t G^{\prime}(t)$, (79) becomes

$$
\begin{aligned}
G_{2}\left(\varepsilon_{2} \chi(s) \mathcal{F}_{2}(s)-\varepsilon_{2} G_{5}(s)\right) \leq & \varepsilon_{2} \chi(s)\left(\mathcal{F}_{2}(s)-\frac{G_{5}(s)}{\chi(s)}\right) G^{\prime}\left(\mathcal{F}_{2}(s)-\frac{G_{5}(s)}{\chi(s)}\right) \\
\leq & \varepsilon_{2} \chi(s) \mathcal{F}_{2}(s) G^{\prime}\left(\mathcal{F}_{2}(s)-\frac{G_{5}(s)}{\chi(s)}\right) \\
& -\varepsilon_{2} \chi(s) \frac{G_{5}(s)}{\chi(s)} G^{\prime}\left(\mathcal{F}_{2}(s)-\frac{G_{5}(s)}{\chi(s)}\right) .
\end{aligned}
$$

Now, using (78) and the increase of $G^{\prime}$, for any $0 \leq s \leq t$, we have

$$
G^{\prime}\left(\mathcal{F}_{2}(s)-\frac{G_{5}(s)}{\chi(s)}\right)<G^{\prime}\left(\mathcal{F}_{2}(s)\right), G^{\prime}\left(\mathcal{F}_{2}(s)-\frac{G_{5}(s)}{\chi(s)}\right)>G^{\prime}\left(\frac{G_{5}(s)}{\chi(s)}\right) .
$$

Combining (81) and (80), we arrive at

$$
G_{2}\left(\epsilon_{1} \chi(s) \mathcal{F}_{2}(s)-\varepsilon_{2} G_{5}(s)\right) \leq \varepsilon_{2} \chi(s) \mathcal{F}_{2}(s) G^{\prime}\left(\mathcal{F}_{2}(s)\right)-\varepsilon_{2} \chi(s) \frac{G_{5}(s)}{\chi(s)} G^{\prime}\left(\frac{G_{5}(s)}{\chi(s)}\right) .
$$

Now we let

$$
\mathcal{F}_{3}(s)=\varepsilon_{2} \chi(s) \mathcal{F}_{2}(s)-\varepsilon_{2} G_{5}(s),
$$

where $\varepsilon_{2}$ is small enough such that $\mathcal{F}_{3}(0) \leq 1$. Recalling the definition of $G_{2},(82)$ becomes, for any $0 \leq s \leq t$,

$$
G_{2}\left(\mathcal{F}_{3}(s)\right) \leq \varepsilon_{2} \chi(t) G_{2}\left(\mathcal{F}_{2}(s)\right)-\varepsilon_{2} \chi(t) G_{2}\left(\frac{G_{5}(s)}{\chi(s)}\right)
$$


Further, we have

$$
\mathcal{F}_{3}^{\prime}(t)=\varepsilon_{2} \chi^{\prime}(t) \mathcal{F}_{2}(t)+\varepsilon_{2} \chi(s) \mathcal{F}_{2}^{\prime}(t)-\varepsilon_{2} G_{5}^{\prime}(t)
$$

Since $\chi^{\prime} \leq 0$, using (76), for any $0 \leq s \leq t$ and $0<\varepsilon_{2} \leq 1$, we obtain

$$
\begin{aligned}
\mathcal{F}_{3}^{\prime}(t) & \leq \varepsilon_{2} \chi(s) \mathcal{F}_{2}^{\prime}(t)-\varepsilon_{2} G_{5}^{\prime}(t) \\
& \leq-c_{1} \varepsilon_{2} \xi(t) \chi(t) G_{2}\left(\mathcal{F}_{2}(t)\right)+c_{2} \varepsilon_{2} \xi(t) \chi(s) G_{4}\left[\frac{c}{d} q(t) h_{2}(t)\right]-\varepsilon_{2} G_{5}^{\prime}(t)
\end{aligned}
$$

Then, using (58) and (84), we get

$$
\begin{aligned}
\mathcal{F}_{3}^{\prime}(t) \leq & -c_{1} \xi(t) G_{2}\left(\mathcal{F}_{3}(t)\right)+c_{2} \varepsilon_{2} \xi(t) \chi(t) G_{4}\left[\frac{c}{d} q(t) h_{2}(t)\right] \\
& -c_{1} \varepsilon_{2} \xi(t) \chi(t) G_{2}\left(\frac{G_{5}(s)}{\chi(s)}\right)-\varepsilon_{2} G_{5}^{\prime}(t) .
\end{aligned}
$$

From the definitions of $G_{1}$ and $G_{5}$ we have

$$
G_{1}\left(G_{5}(s)\right)=c_{1} \int_{0}^{s} \xi(\tau) d \tau
$$

and hence

$$
G_{5}^{\prime}(s)=-c_{1} \xi(s) G_{2}\left(G_{5}(s)\right)
$$

Now we have

$$
\begin{gathered}
c_{2} \varepsilon_{2} \xi(t) \chi(t) G_{4}\left[\frac{c}{d} q(t) h_{2}(t)\right]-c_{1} \varepsilon_{2} \xi(t) \chi(t) G_{2}\left(\frac{G_{5}(s)}{\chi(s)}\right)-\varepsilon_{2} G_{5}^{\prime}(t) \\
=c_{2} \varepsilon_{2} \xi(t) \chi(t) G_{4}\left[\frac{c}{d} q(t) h_{2}(t)\right]-c_{1} \varepsilon_{2} \xi(t) \chi(t) G_{2}\left(\frac{G_{5}(s)}{\chi(s)}\right)+c \varepsilon_{2} \xi(t) G_{2}\left(G_{5}(t)\right) \\
=\varepsilon_{2} \xi(t) \chi(t)\left(c_{2} G_{4}\left[\frac{c}{d} q(t) h_{2}(t)\right]-c_{1} G_{2}\left(\frac{G_{5}(s)}{\chi(s)}\right)\right)+\frac{G_{2}\left(G_{5}(t)\right)}{\chi(t)} .
\end{gathered}
$$

Then, according to (58), we get

$$
\varepsilon_{2} \xi(t) \chi(t)\left(c_{2} G_{4}\left[\frac{c}{d} q(t) h_{2}(t)\right]-c_{1} G_{2}\left(\frac{G_{5}(s)}{\chi(s)}\right)\right)-\frac{G_{2}\left(G_{5}(t)\right)}{\chi(t)} \leq 0
$$

Then (87) gives

$$
\mathcal{F}_{3}^{\prime}(t) \leq-c_{1} \xi(t) G_{2}\left(\mathcal{F}_{3}(t)\right)
$$

Thus from (90) and the definitions of $G_{1}$ and $G_{2}$ in (55) and (56) we obtain

$$
\left(G_{1}\left(\mathcal{F}_{3}(t)\right)\right)^{\prime} \geq c_{1} \xi(t)
$$


Integrating (91) over $[0, t]$, we get

$$
G_{1}\left(\mathcal{F}_{3}(t)\right) \geq c_{1} \int_{0}^{t} \xi(s) d s+G_{1}\left(\mathcal{F}_{3}(0)\right)
$$

Since $G_{1}$ is decreasing, $\mathcal{F}_{3}(0) \leq 1$, and $G_{1}(1)=0$, we have

$$
\mathcal{F}_{3}(t) \leq G_{1}^{-1}\left(c_{1} \int_{0}^{t} \xi(s) d s\right)=G_{5}(t) .
$$

Recalling that $\mathcal{F}_{3}(t)=\varepsilon_{2} \chi(t) \mathcal{F}_{2}(t)-\varepsilon_{2} G_{5}(t)$, we have

$$
\mathcal{F}_{2}(t) \leq \frac{\left(1+\varepsilon_{2}\right)}{\varepsilon_{2}} \frac{G_{5}(t)}{\chi(t)}
$$

Similarly, recalling that $\mathcal{F}_{2}(t):=d_{0} \mathcal{F}_{1}(t) q(t)$, we get

$$
\mathcal{F}_{1}(t) \leq \frac{\left(1+\varepsilon_{2}\right)}{d_{0} \varepsilon_{2}} \frac{G_{5}(t)}{\chi(t) q(t)}
$$

Since $\mathcal{F}_{1} \sim E$, for some $b>0$, we have $E(t) \leq b \mathcal{F}_{1}$, which gives

$$
E(t) \leq \frac{b\left(1+\varepsilon_{2}\right)}{d_{0} \varepsilon_{2}} \frac{G_{5}(t)}{\chi(t) q(t)}
$$

From (77) and (96) we obtain the estimate

$$
E(t) \leq c_{3}\left(\frac{G_{5}(t)}{\chi(t) q(t)}\right)
$$

where $c_{3}=\max \left\{\frac{2}{b_{0}}, \frac{b\left(1+\varepsilon_{2}\right)}{d_{0} \varepsilon_{2}}\right\}$.

In the following example, we illustrate our decay result.

Example 4.2 Let $h(t)=\frac{a}{(1+t)^{v}}$, where $v>1$ and $0<a<v-1$, so that $(A 1)$ is satisfied. In this case, $\xi(t)=v a^{\frac{-1}{v}}, H(t)=t^{\frac{v+1}{v}}$, and $G^{-1}(t)=\left(H^{-1}(t)\right)^{\frac{1}{1+\varepsilon_{0}}}$. Then for any $\varepsilon_{0} \in(0,1)$, we have $G(t)=t^{\lambda}$, where $\lambda:=\frac{\left(\varepsilon_{0}+1\right)(v+1)}{v}>1$. Recall the definitions of the functions $G_{i}, i=1, \ldots, 5$ :

$$
\begin{aligned}
& G_{1}(t)=a_{1}\left(t^{1-\lambda}-1\right), \quad G_{2}(t)=a_{2} t^{\lambda}, \quad G_{3}(t)=a_{3} t^{\frac{\lambda}{\lambda-1}}, \quad G_{4}(t)=a_{4} t^{\lambda} \\
& G_{5}(t)=a_{5}(1+t)^{\frac{1}{1-\lambda}}
\end{aligned}
$$

where $a_{i}, i=1,2,3,4,5$, are positive constants depending on $a, v$, and $\varepsilon_{0}$. As in [30], we consider

$$
m_{0}(1+t)^{r} \leq 1+\left\|\Delta u_{0}\right\|^{2} \leq m_{1}(1+t)^{r}
$$

where $r<v-1$ and $m_{0}, m_{1}>0$. Then for some positive constants $a_{i}(i=6,7)$ depending only on $a, v, m_{0}, m_{1}, r$, we have

$$
a_{6}(1+t)^{-v+1+r} \leq h_{1}(t) \leq a_{7}(1+t)^{-v+1+r},
$$


where $-v+1+r<0$. Recalling the definitions of the functions $h_{1}, h_{2}$, and $q$, we have

$$
q(t) h_{2}(t)=(1+t)^{\frac{-v+r}{1+\varepsilon_{0}}}
$$

It is clear that condition (58) is satisfied if

$$
q^{\lambda}(t) h_{2}^{\lambda}(t) \chi^{\lambda}(t)+(1+t)^{\frac{-\lambda}{\lambda-1}} \chi^{\lambda-1}(t) \leq(1+t)^{\frac{-\lambda}{\lambda-1}}
$$

Choosing $\chi(t)=(1+t)^{m}$, where $m<\min \left(0, \frac{-1}{\lambda-1}+\frac{(\nu-r)(v+1)}{\lambda v}\right)$, we have the following two cases depending on $r$.

Case 1: If $0<r<v-1$, then for any $\varepsilon>0$, there exists $C_{\varepsilon}>0$ such that we have the following decay rate estimate of $E(60)$ :

$$
E(t) \leq C_{\varepsilon}(1+t)^{-(v-r-1)+\varepsilon} .
$$

Case 2: If $r \leq 0$, then for any $\varepsilon>0$, there exists $C_{\varepsilon}>0$ such that the decay rate estimate of $E(60)$ is given by:

$$
E(t) \leq C_{\varepsilon}(1+t)^{-(v-1)+\varepsilon} .
$$

Thus estimates (102) and (103) give $\lim _{t \rightarrow+\infty} E(T)=0$.

\section{Conclusion}

As far as we know, there are no decay results in the literature known for logarithmic plate equation with infinite memory and a wider class of relaxation functions. Our work extends the works for some wave equations treated in the literature to the plate equation with logarithmic nonlinearity. Also, we succeed to extend some general decay results, known for the case of finite history, to the case of infinite history, where the relaxation function satisfies a wider class of relaxation functions. Furthermore, we dropped the boundedness assumption on the history data considered in earlier results in the literature.

Acknowledgements

The author thanks KFUPM for its continuous support and an anonymous referee for her/his careful reading and valuables remarks. This work is funded by KFUPM under Project (SB181039).

Funding

This work is funded by KFUPM under Project (SB181039).

Abbreviations

Not applicable.

Availability of data and materials

Not applicable.

Competing interests

The author declares that he has no competing interests.

Authors' contributions

I read and approved the final manuscript.

Authors' information

Not applicable. 


\section{Publisher's Note}

Springer Nature remains neutral with regard to jurisdictional claims in published maps and institutional affiliations.

\section{Received: 5 January 2020 Accepted: 22 April 2020 Published online: 01 May 2020}

\section{References}

1. Gorka, P.: Logarithmic Klein-Gordon equation. Acta Phys. Pol. 40, 59-66 (2009)

2. Bartkowski, K., Górka, P.: One-dimensional Klein-Gordon equation with logarithmic nonlinearities. J. Phys. A, Math. Theor. 41(35), Article ID 355201 (2008)

3. Hiramatsu, T., Kawasaki, M., Takahashi, F.: Numerical study of Q-ball formation in gravity mediation. J. Cosmol. Astropart. Phys. 2010(6), Article ID 008 (2010)

4. Bialynicki-Birula, I., Mycielski, J.: Wave equations with logarithmic nonlinearities. Bull. Acad. Pol. Sci., Cl. Trois. 23, 461-466 (1975)

5. Bialynicki-Birula, I., Mycielski, J.: Nonlinear wave mechanics. Ann. Phys. 100(1-2), 62-93 (1976)

6. Cazenave, T., Haraux, A.: Équations d'évolution avec non linéarité logarithmique. Ann. Fac. Sci. Toulouse, 2, 21-51 (1980)

7. Al-Gharabli, M.M., Guesmia, A., Messaoudi, S.A.: Existence and a general decay results for a viscoelastic plate equation with a logarithmic nonlinearity. Commun. Pure Appl. Anal. 18(1), 159-180 (2019)

8. Al-Gharabli, M.M., Guesmia, A., Messaoudi, S.A.: Well-posedness and asymptotic stability results for a viscoelastic plate equation with a logarithmic nonlinearity. Appl. Anal. 99(1), 50-74 (2018)

9. Al-Gharabli, M.M.: New general decay results for a viscoelastic plate equation with a logarithmic nonlinearity. Bound. Value Probl. 2019, Article ID 194 (2019)

10. Han, X.: Global existence of weak solutions for a logarithmic wave equation arising from Q-ball dynamics. Bull. Korean Math. Soc. 50(1), 275-283 (2013)

11. Lian, W., Xu, R.: Global well-posedness of nonlinear wave equation with weak and strong damping terms and logarithmic source term. Adv. Nonlinear Anal. 9(1), 613-632 (2019)

12. Peyravi, A.: General stability and exponential growth for a class of semi-linear wave equations with logarithmic source and memory terms. Appl. Math. Optim. 81, 545-561 (2020)

13. Xu, R., Lian, W., Kong, X., Yang, Y.: Fourth order wave equation with nonlinear strain and logarithmic nonlinearity. Appl. Numer. Math. 141, 185-205 (2019)

14. Wang, X., Chen, Y., Yang, Y., Li, J., Xu, R.: Kirchhoff-type system with linear weak damping and logarithmic nonlinearities. Nonlinear Anal. 188, 475-499 (2019)

15. Giorgi, C., Rivera, J.E.M., Pata, V.: Global attractors for a semilinear hyperbolic equation in viscoelasticity. J. Math. Anal. Appl. 260(1), 83-99 (2001)

16. Conti, M., Pata, V.: Weakly dissipative semilinear equations of viscoelasticity. Commun. Pure Appl. Anal. 4(4), 705-720 (2005)

17. Appleby, J.A., Fabrizio, M., Lazzari, B., Reynolds, D.W.: On exponential asymptotic stability in linear viscoelasticity. Math. Models Methods Appl. Sci. 16(10), 1677-1694 (2006)

18. Pata, V.: Stability and exponential stability in linear viscoelasticity. Milan J. Math. 77(1), 333-360 (2009)

19. Guesmia, A.: Asymptotic stability of abstract dissipative systems with infinite memory. J. Math. Anal. Appl. 382(2), 748-760 (2011)

20. Guesmia, A., Messaoudi, S.A.: A general decay result for a viscoelastic equation in the presence of past and finite history memories. Nonlinear Anal., Real World Appl. 13(1), 476-485 (2012)

21. Al-Mahdi, A.M., Al-Gharabli, M.M.: New general decay results in an infinite memory viscoelastic problem with nonlinear damping. Bound. Value Probl. 2019, Article ID 140 (2019)

22. Guesmia, A., Messaoudi, S.A.: A new approach to the stability of an abstract system in the presence of infinite history. J. Math. Anal. Appl. 416(1), 212-228 (2014)

23. Alabau-Boussouira, F., Cannarsa, P.: A general method for proving sharp energy decay rates for memory-dissipative evolution equations. C. R. Math. 347(15-16), 867-872 (2009)

24. Cavalcanti, M.M., Cavalcanti, A.D., Lasiecka, I., Wang, X.: Existence and sharp decay rate estimates for a von Karman system with long memory. Nonlinear Anal., Real World Appl. 22, 289-306 (2015)

25. Yang, X.-G., Zhang, J., Wang, S.: Stability and dynamics of a weak viscoelastic system with memory and nonlinear time-varying delay. Discrete Contin. Dyn. Syst., Ser. A 40(3), 1493-1515 (2020)

26. Conti, M., Pata, V.: General decay properties of abstract linear viscoelasticity. Z. Angew. Math. Phys. 71, Article ID 6 (2020)

27. Al-Mahdi, A.M.: Optimal decay result for Kirchhoff plate equations with nonlinear damping and very general type of relaxation functions. Bound. Value Probl. 2019, Article ID 82 (2019)

28. Mustafa, M.I., Messaoudi, S.A.: General stability result for viscoelastic wave equations. J. Math. Phys. 53(5), Article ID $053702(2012)$

29. Mustafa, M...: Optimal decay rates for the viscoelastic wave equation. Math. Methods Appl. Sci. 41(1), 192-204 (2018)

30. Guesmia, A.: New general decay rates of solutions for two viscoelastic wave equations with infinite memory. Math. Model. Anal. (2020, in press)

31. Messaoudi, S.A., Al-Khulaifi, W.: General and optimal decay for a quasilinear viscoelastic equation. Appl. Math. Lett. 66 , 16-22 (2017)

32. Gross, L.: Logarithmic Sobolev inequalities. Am. J. Math. 97(4), 1061-1083 (1975)

33. Chen, H., Luo, P., Liu, G.: Global solution and blow-up of a semilinear heat equation with logarithmic nonlinearity. J. Math. Anal. Appl. 422(1), 84-98 (2015)

34. Arnold, V.I: Mathematical Methods of Classical Mechanics. Graduate Texts in Mathematics, vol. 60. Springer, Berlin (2013) 